\title{
Es tiempo de estimular la innovación y el desarrollo de tecnología intervencionista en el CACl
}

\author{
It's time to stimulate innovation and development of interventional \\ technology in the $\mathrm{CACl}$
}

Revista Argentina de Cardioangiología Intervencionista 2018;9(4):37. DOI: 10.30567/RACI/201804/0037-0037

\begin{abstract}
En esta nueva edición de la Revista Argentina de Cardioangiología Intervencionista, quiero compartir con ustedes una iniciativa que está madurando de la mano de socios de nuestro Colegio, y que se refiere a un aspecto relevante en cuanto a la proyección futura del CACI.

No caben dudas de la madurez que ha alcanzado nuestro Colegio en las áreas de docencia y educación continua, expresadas claramente por el prestigio y reconocimiento de la Carrera UBA-CACI, el Programa de Actualización y ratificada en el último año con el lanzamiento e inauguración de nuevas herramientas educativas, tales como el ebook de Cardioangiología Intervencionista y el Centro de Simulación CACI. De la misma forma, la proyección del Colegio en la actividad científica a través de su participación con sesiones en los más prestigiosos congresos internacionales de la especialidad y en congresos nacionales es indiscutible. También es reconocido el rol del CACI en aspectos relacionados a la seguridad y calidad de la práctica de nuestra especialidad.

En este contexto, la iniciativa a la que me refiero es la creación de un centro, secretaría o agencia de Innovación y desarrollo tecnológico en cardioangiología intervencionista del CACI.

En sus comienzos, los socios fundadores del CACI debieron invertir su tiempo en crear y fortalecer al Colegio y la especialidad. En una segunda etapa, los objetivos y esfuerzos de las nuevas comisiones directivas apuntaron a los aspectos anteriormente mencionados, de docencia, educación continua, visibilidad nacional e internacional del CACI a través de la actividad científica y la seguridad y calidad de la práctica.

Es el momento para invertir el tiempo y el esfuerzo necesarios para expandir los objetivos del CACI en la actividad de ciencia y técnica, con el deseo de ver engrandecer, desde el Colegio, a nuestro país, que tanto necesita de nuestro esfuerzo e inteligencia para su reconstrucción.

Esta secretaría o agencia de Innovación del CACI la imagino como la construcción de un edificio desde la base, que tiene que ser muy sólida, que cobre vida propia y tenga caraterísticas únicas, y que respaldado por distintos entes viva por sus propias metas, con reglamento, finalidades y actividad propia. Es necesaria una infraestructura dedicada. La idea es fomentar la investigación. Hay que llegar a la situación que emprendedores, startups, etc., tengan en el CACI el referente obligado para el desarrollo de iniciativas y financiamiento por parte de inversores en el mundo de la tecnología médica.
\end{abstract}

Los objetivos básicos de esta nueva secretaría o agencia serían:

1. Acompañar a los cardioangiólogos intervencionistas emprendedores en el desarrollo de proyectos de innovación.

2. Proporcionar el soporte para subir los peldaños del proyecto.

3. Ofrecer los instrumentos orientados a estos propósitos.

4. Promover capacidades tecnológicas y de investigación en innovación, calificadas.

5. Crear lo que podríamos llamar un ecosistema de innovación, cooperación y asistencia tecnológica.

6. Estimular los proyectos de investigación preclínica, first in man y clínica de nuevos dispositivos en Argentina.

7. Formación de recursos humanos orientados a la innovación, dentro de las nuevas generaciones de cardioangiólogos intervencionistas.

Se trata, de un proyecto ambicioso, a largo plazo y progresivo, que requiere la convocatoria de voluntades dispuestas a plantar la semilla, para que el proyecto germine con el tiempo. Proyectaría al CACI a otro nivel y, con el esfuerzo de todos, podríamos generar la cultura en las nuevas y futuras generaciones de cardioangiólogos intervencionistas, en el desarrollo de la investigación e innovación tecnológica relacionada a nuestra especialidad.

Los invito a que enfrentemos juntos desde el CACI, este nuevo desafío. 